\title{
Clinical features, predictive correlates, and pathophysiology of immune-related adverse events in immune checkpoint inhibitor treatments in cancer: a short review
}

\author{
This article was published in the following Dove Press journal: \\ ImmunoTargets and Therapy \\ 10 October 2017 \\ Number of times this article has been viewed
}

\author{
Jennifer M Yoest \\ Department of Pathology, University \\ of Pittsburgh Medical Center, \\ Pittsburgh, PA, USA
}

\begin{abstract}
Identification and characterization of T-cell regulatory mechanisms, or checkpoints, have led to a wave of drug development aimed at inhibiting these targets to "remove the brakes" of the immune system. This class of anticancer therapeutics, termed immune checkpoint inhibitors (ICIs), has harnessed the potential of the body's own immune system to recognize cancerous cells and selectively eliminate them, in some cases with alarming success. This new breakthrough, however, has not been without its drawbacks. Immune-related adverse events (irAEs) are adverse events encountered during treatment with ICIs that are thought to be mediated through the patient's immune system which can manifest with a variety of symptoms which often resemble autoimmunity. These events range widely in presentation and severity and are reported frequently. Here, we will discuss a large selection of case reports in order to inform the clinician, laboratorian, and researcher of the scope of organ systems affected, the severity of the conditions being encountered, and the responses of these events to treatment, as well as explore the use of ICIs in the setting of preexisting autoimmunity. We will also consider the ability to detect autoantibodies before and during irAEs as well as the correlations that irAEs have with clinical outcomes. Finally, we will conclude by exploring the possibility that two distinct pathways may be contributing to the phenomenon of irAEs within this class of drugs, and the role that this might play in future research and clinical practice.
\end{abstract}

Keywords: immune checkpoint inhibitors, immune-related adverse events, side effects, autoimmunity, etiology, prediction, cross-reactive, correlation with tumor response

\section{Background}

The human immune system is a powerful tool which is able to detect both exogenous and endogenous threats, including signs of malignancy. Therefore, it has long been a goal of science and medicine to be able to harness the power of the body's own immune system to fight cancer. One rapidly growing approach to this problem has been to modulate immune regulatory mechanisms in order to augment an antitumor response. T-cell co-receptors present a potential target for drug development, as they can either promote or downregulate T-cell activation. Two inhibitory T-cell co-receptors, cytotoxic T-lymphocyte antigen 4 (CTLA-4) and programmed cell death 1 (PD-1), along with its ligands programmed cell death ligand 1 (PD-L1) and 2, function through two independent signaling pathways to downregulate the immune system. ${ }^{1-3}$ Normally, these regulatory receptors, or checkpoints, serve to retain balance between activity and quiescence in the immune system. Research has shown that CTLA-4 is involved in thymic
Correspondence: Jennifer M Yoest Department of Pathology, University of Pittsburgh Medical Center, A7I I Scaife Hall, 3550 Terrace Street, Pittsburgh, PA I5213, USA

Tel + I 4128026013

Fax + I 4128026079

Email yoestjm@upmc.edu 
T-cell maturation and peripheral inhibition or downregulation of T-cell activation, while PD-1 has been demonstrated to be directly involved in the peripheral tolerance of self-reactive T-cells which escape thymic deletion. ${ }^{4-6}$ It is hypothesized that tumors effectively escape immune detection through strategies broadly termed "adaptive immune resistance", which is an area of active investigation. One such strategy involves inducing quiescence in tumor-reactive cytotoxic T-lymphocytes through the use of these inhibitory pathways. ${ }^{7}$ The related concept of T-cell exhaustion, mediated through these and other immune checkpoints, is theorized to play a role in immune quiescence in chronic infectious diseases. The interested reader is directed to two excellent recent reviews of the immune checkpoint pathways and the interplay with the microenvironment in both malignancy and chronic infectious diseases by Dyck and Mills and by Rao et al., ${ }^{2,3}$ Blocking these checkpoints via monoclonal antibodies designed to bind these regulatory receptors or their intended ligands "removes the brakes" on the immune system and has been proven to be effective with varying degrees of success in several primary tumor types. Five drugs in the immune checkpoint inhibitor (ICI) class have been approved for use against various malignancies to date, while work on the chronic infectious diseases front, although promising, has been largely preclinical. ${ }^{2,3,8,9}$ Currently, approved ICIs include the human IgG1 monoclonal antibody ipilimumab which targets CTLA-4, the humanized and fully human IgG4 monoclonal antibodies pembrolizumab and nivolumab which each target PD-1, the humanized IgG1 monoclonal antibody atezolizumab targeting PD-L1, and the recently approved human IgG1 monoclonal antibodies durvalumab and avelumab, which also target PD-L1. These drugs and many others which also target immune checkpoints are under ongoing investigation for expansion of their use to include a vast array of primary tumors, and in a landmark move by the US Food and Drug Administration (FDA), pembrolizumab has recently been approved for use in any solid tumor demonstrating a microsatellite instability-high or mismatch-repair-deficient genetic signature, thereby expanding the availability of ICIs to many more patients..$^{9,10}$

\section{Immune-related adverse events (irAEs)}

Immune checkpoints are necessary for maintaining balance between activation and quiescence of the immune system and to suppress self-reactive T-cells. By blocking these checkpoints, ICIs disrupt this delicate balance and can lead to autoimmune-like events. This effect was first described in animal models of immune checkpoint blockade. For instance, CTLA-4-deleted mice developed T-cell dysregulation and demonstrated tissue damage such as myocarditis and pancreatitis, and PD-1-deleted mice developed autoimmune diseases such as arthritis, glomerulonephritis, and autoantibody-positive dilated cardiomyopathy., ${ }^{41,12}$ Autoimmune-like side effects were first observed in humans during trials of ICIs targeting CTLA- 4 and PD- 1 and were termed irAEs. irAEs were encountered in early clinical trials of all ICIs and most frequently included colitis, interstitial pneumonitis, rash or other dermatologic findings, transaminitis, and endocrinopathies, although many other irAEs were described at much lower frequencies. ${ }^{13-21}$ The reported rate of adverse events of any grade and any etiology related to treatment was $\sim 70 \%-90 \%$ in these Phase I and II trials, but the majority of these adverse events were reported to be of grade 1 or 2 with grade 3 or higher irAEs occurring in up to $18 \%$ of patients. ${ }^{13-19}$

It can be difficult to compare rates of irAEs across clinical trials due to several factors. Foremost among these is the different classification criteria for adverse events used across different trials. Some trials described "treatment-related" events while others described "drug-related" events, and still others specifically described "immune-related" or "immunemediated" events, which could either be individually investigator-determined or predetermined by a list of specific events of interest provided to ancillary investigators by the primary research team. A few trials classified events into more than one of these categories, with the overlap difficult to tease apart. Although raw data on adverse events were available in some texts and supplemental material, the fact that multiple adverse events could be reported in a single patient further complicates the interpretation of these data. Furthermore, trials which included very few patients were underpowered to detect infrequent events. Finally, the follow-up period and treatment doses both varied within and between trials which could have affected the rates of detection of irAEs among trials. Day and Hansen highlight some of these concerns in a recent review of irAE rate reporting in the literature. ${ }^{20}$

With these challenges in mind, the rates of irAEs of any grade described in early Phase I and II trials of ipilimumab, nivolumab, and pembrolizumab were reported to be anywhere from $10 \%$ to $80 \%$. irAEs of grade 3 or higher, however, were reported in only $2.5 \%-18 \%$ of participants. ${ }^{13-19}$ Day and Hansen report similar numbers in their recent and thorough review of larger Phase III trials, with a majority of patients receiving ICIs reporting some type of treatmentrelated event (ranging from $58 \%$ to $96 \%$ of participants), 
but with substantially fewer grade 3 or higher adverse events (7\%-59\%). We can also see more clearly from their review of the literature that irAE rates are higher for CTLA-4 blockade than with PD-1 blockade. ${ }^{20}$ Rates of grade 3 or 4 drug-related adverse events also appear to be much higher for combinations of CTLA-4 and PD-1 blockade than for either of these classes given separately, with Phase I trials reporting these events in 53\%-54\% of participants as compared to $18 \%-24 \%$ of patients in these same trials who received only anti-PD-1 treatment; similar rates have been seen in other combination trials. ${ }^{20-24}$ The difference in rates of irAEs that are reported between separate ICI agents is best explained by the different points in the T-cell activation process that the immune checkpoint targets occupy. That combination therapy targeting more than one checkpoint pathway would have a higher rate of irAEs seems to follow logically.

Atezolizumab, durvalumab, and avelumab are the newest ICIs to be approved by the FDA, but individual case reports of severe events have already emerged in the literature..$^{25-27}$ Rates of irAEs of grade 3-5 range from 1\% to 9\% across several Phase I and II studies released recently and appear to be lower than for other drugs in the ICI class, although this comparison also suffers from the limitations discussed above. ${ }^{28-35}$ It will be important to revisit and confirm the irAE rates for these newer ICIs when data from larger Phase III trials become available.

Despite the wide range of reported rates and organ systems affected, the vast majority of adverse events attributed to immune-related etiologies were described as grade 1 or 2 and resolved with systemic corticosteroid treatment. Rarely, specific irAEs were described as requiring tumor necrosis factor (TNF)-alpha inhibiting agents or surgery for resolution (as in cases of severe or perforated colitis), or as being the direct cause of a participant's death. ${ }^{13,15,16}$ Since approval by the FDA of ICIs for use in various malignancies and at various lines of defense, independent case reports and characterizations of infrequent and severe irAEs have steadily streamed into the literature, and with them speculation as to the exact pathogenesis of these events, their predictability, and their correlation with tumor response. The discussion that follows will explore the various presentations of these events including the organ systems affected, the severity of events and their response to treatment, and autoantibody detection during and before irAEs. We will also briefly explore ICI treatment in patients with preexisting autoimmunity, the correlation between irAEs and tumor response, and finally, we will revisit the etiology of these adverse events in light of the evidence explored here.

\section{Organ systems affected and timing of events}

The first feature of irAEs which is important to recognize is their indiscriminate nature. Mild to moderate irAEs of certain organ systems like the gastrointestinal tract, lungs, endocrine system, and skin, and nonspecific findings such as fatigue, were seen most often in initial trials of ICIs; severe events were seen only occasionally. ${ }^{13-19}$ Case reports and case series outside of clinical trials have described some of the relatively more common dermatologic, gastrointestinal, and endocrine events, but case reports have also emerged detailing severe involvement of nearly every organ system and at every point in treatment. Onset of events has been described as close to initiation of ICI therapy as 8 days, and as remote as $>1$ year, and the organ systems involved have included the central nervous system (CNS), cardiovascular system, musculoskeletal system, hepatic and renal systems, and even include myasthenia gravis, hemolytic anemia, sarcoidosis, and solid-organ transplant rejection. ${ }^{25-27,36-80}$ Therefore, awareness of only the initial, commonly reported cases or a selection of scattered independent case reports is not sufficient to prepare clinicians for the immediate and long-term surveillance for these events that is required to effectively mitigate these toxicities among their patients.

\section{Severity of events and response to treatment}

In addition to the vast heterogeneity of the organ systems affected and the timing of onset is the wide range of severity of these adverse events and their varied responsiveness to treatment. According to clinical trials of ipilimumab, pembrolizumab, and nivolumab, a majority of the observed irAEs were successfully treated with systemic corticosteroids, but this was not always the case. ${ }^{13-19}$ In a clinical trial for a combination of ipilimumab and nivolumab in melanoma patients, $38 \%$ of patients with treatment-related adverse events received systemic glucocorticoids and $4 \%$ of patients experiencing a treatment-related adverse event required additional immunomodulators such as infliximab or mycophenolate mofetil..$^{22}$ In a retrospective analysis of ICI-treated patients at their institution, Cappelli et al identified 13 patients who developed rheumatologic irAEs specifically. They went on to describe in detail the treatment required for each patient's inflammatory arthritis, sicca syndrome, or additional irAEs to enter remission. Many of these patients experienced resolution of one or more irAEs with various dosages and durations of corticosteroid treatment. However, four of these patients 
required additional treatment for remission of either colitis or inflammatory arthritis, including methotrexate, adalimumab, infliximab, and/or etanercept. ${ }^{49}$ Hofmann et al retrospectively reviewed irAEs due to nivolumab or pembrolizumab treatment reported across 15 centers in Germany and Switzerland noting that some of these events resolved without treatment, some events resolved or improved with corticosteroid or other treatment, and some events did not resolve. Of note, the events which did not require intervention were most commonly reported to be dermatologic in nature. ${ }^{50}$ Horvat et al describe their experience with irAEs in patients on clinical trials of ICIs at their institution and observed that $\sim 10 \%$ of those treated with systemic corticosteroids for an irAE did not experience adequate resolution. ${ }^{51}$ The group recommend, based on their observations, that the threshold for initiating systemic corticosteroids should be low, and further that the threshold for escalating treatment to an anti-TNF-alpha agent should be considered as soon as a week into treatment with high-dose corticosteroids if symptomatic improvement is not seen.

\section{Irreversible events}

At the more extreme end of the spectrum, there have been several reports of irAEs which have resulted in irreversible morbidities. Many individual cases and case series have been published thus far describing the irreversible nature of irAEs which have affected the endocrine system. In particular, the acute onset of diabetic ketoacidosis with resultant insulindependent diabetes mellitus has been described in several patients. ${ }^{26,50,52-55}$ In addition, primary adrenal failure requiring hydrocortisone and fludrocortisone replacement has also been described. ${ }^{56}$ Ryder et al describe, in a retrospective review of primary clinical trial patients receiving ipilimumab or ipilimumab and nivolumab simultaneously at their institution, that the majority of observed endocrine irAEs (excluding thyroid dysfunction) did not recover endogenous hormone secretion. ${ }^{57}$ Hofmann et al noted 25 of the 33 reported cases of endocrinopathies related to nivolumab or pembrolizumab treatment from their multicenter review did not resolve after treatment discontinuation, immunosuppression, and/or hormone replacement. These events included hypothyroidism, hyperthyroidism, hypopituitarism, and insulin-dependent diabetes mellitus. ${ }^{50}$ From the above investigations, it would appear that irAE of the endocrine system may result in irreversible damage or destruction of the tissues of these organs and often requires not only immunosuppressive treatment, but also permanent hormone replacement therapy. Other non-resolving irAEs reported in Hofmann et al's retrospective analysis included hepatitis, pancreatic insufficiency following pancreatitis, diarrhea, xerostomia, alopecia, and vitiligo. ${ }^{50}$ Colitis, which was more often observed with ipilimumab than with other ICIs, occasionally necessitated colectomy, in the setting of either perforation or incomplete response to medical management. ${ }^{15,81}$

Reports detailing the experience of solid-organ transplant recipients who receive ICI as a last-line treatment for malignancies have emerged revealing swift and irreversible graft dysfunction in some cases, while in other cases dysfunction is either reversed or not observed at all. ${ }^{58-64}$ With the small number of cases reported thus far it is difficult to draw concrete conclusions, although it appears that the risk of graft rejection may be higher with PD-1 blockade as compared with CTLA-4 blockade. ${ }^{61}$ Anecdotal evidence suggests that co-administration of low-dose corticosteroids and mTOR inhibitors during ICI treatment may improve graft survival, and further investigation into this possibility is warranted. ${ }^{64}$ Therefore, the consequences of irreversible graft dysfunction or loss should be carefully considered before treatment with ICIs in solid-organ transplant recipients.

\section{Fatalities}

Several reported fatalities have been directly attributed to ICIassociated irAEs and have involved a range of organ systems. Autoantibody-positive autoimmune myositis was found to be responsible for rhabdomyolysis and third-degree atrioventricular block in one patient who ultimately succumbed to multiorgan failure and likely pneumonia just 26 days after starting nivolumab. ${ }^{65}$ Myocarditis was the suspected cause of death in another patient who had a history of Wolff-ParkinsonWhite syndrome. In this case, Gibson et al reported that the onset of symptoms in their patient started 1 week after the second dose of nivolumab and ultimately culminated in elevated cardiac enzymes, electrocardiogram abnormalities, and ventricular tachycardia despite no history of atherosclerotic cardiovascular disease and no visible motion abnormalities on echocardiogram. ${ }^{66}$ Autoimmune hemolytic anemia was discovered by laboratory workup to be the cause of a profound and eventually fatal case of anemia which became symptomatic $<3$ weeks after nivolumab initiation. ${ }^{67} \mathrm{~A}$ fatality due to interstitial pneumonitis and acute respiratory distress syndrome was reported by Nishino et al, along with two cases of pneumonitis that resolved with treatment. ${ }^{68} \mathrm{~A}$ case of fatal CNS demyelinating disease was confirmed on autopsy in a patient who began showing nonspecific symptoms $<2$ months after starting nivolumab treatment secondary to failure of ipilimumab therapy. Although this patient was described as 
initially improving with corticosteroids, intravenous immunoglobulin (IVIG), and time, his mental status declined again several months later and IVIG treatment proved ineffective. ${ }^{69}$ At least three patients have also been described with fatal fulminant myasthenia gravis, two of which presented $<3$ weeks after initiation of ICI treatment and expired just days to weeks later. ${ }^{70,71}$ Another fatal myasthenia gravis case was noted during a Phase Ib trial of combination CTLA-4 and PD-1 blockade with durvalumab and tremelimumab, along with two other suspected treatment-related deaths that were not described in detail. ${ }^{23}$ Other ICI trials have reported potential treatment-related deaths, but details are not specific enough to discern the exact etiologies of these events. . $^{13,17,18,34}$

\section{Detectable autoantibodies}

In a few instances, reported investigations of irAEs have included analysis of clinically relevant autoantibodies with some very interesting results. Various autoantibodies have been detected after an irAE in patients who have a known history of autoimmunity, but autoantibodies have also been detected subsequent to an event in cases where the patient has no clinical history of autoimmunity. ${ }^{49,54,55,65,70,72,73}$ In one case series and in one independent report, four of six patients who experienced acute-onset insulin-dependent diabetes mellitus were found to be positive for one or more well-described type 1 diabetes mellitus-specific autoantibodies including insulin autoantibody, glutamic acid decarboxylase autoantibody, and anti-islet cell autoantigen (also known as islet antigen 2). ${ }^{54,55}$ These cases demonstrated precipitous onset of autoimmune type 1 diabetes with diabetic ketoacidosis and detectable autoantibodies in patients aged $>55$. Several patients with clinical signs and symptoms of myasthenia gravis following ICI treatment have been found to be positive for myasthenia gravis-associated autoantibodies. Multiple patients tested positive for anti-acetylcholine receptor antibodies (AChR) and at least one case described positivity for AChR blocking antibodies, AChR modulating antibodies, and anti-striated muscle (striational) antibodies all in the same patient. ${ }^{34,70,71,74}$ A range of other positive, specific and nonspecific autoantibody findings have also been described including antinuclear antibodies, anti-Ro/SSA, anti-La/SSB, rheumatoid factor, and others. ${ }^{49,75}$

Detection of autoantibodies during an adverse event would confirm an immune-mediated etiology of the event in question, but unfortunately this approach appears to lack sensitivity. Whether this reflects the underlying mechanism of the event, an inability to assay an as-yet undescribed autoantibody, or simply an inability to detect a very low level of the specific autoantibody involved is not known. In some cases, however, subclinical levels of autoantibodies have been detected in pretreatment samples from patients who went on to develop an autoantibody-positive irAE. ${ }^{34,70,73}$ Of course, not all cases of irAEs with detectable autoantibody positivity had pretreatment samples available for testing, and even when available, it appears that very few patients with specific, corresponding, and detectable autoantibodies after an irAE were also found to be low-level positive before treatment. The existence of cases where positivity for low levels of autoantibodies is discovered prior to ICI treatment and which subsequently develop a correlating irAE supports the theory that ICIs remove peripheral tolerance of preexisting self-reactive T-cells. The detection of specific autoantibodies before treatment and after treatment and their significance in relationship to any irAEs which develop is an area of considerable interest which may prove valuable in irAE prediction and merits further investigation.

\section{Preexisting autoimmunity}

Another facet of the discussion on irAEs which warrants mention here is the issue of ICI treatment in patients with a history of autoimmunity. Patients with known autoimmunity were excluded from clinical trials of ICIs, ostensibly for fear of autoimmune exacerbations and unacceptable toxicity among these patients based on the presumed overlap between the mechanism of action of the ICI class of drugs and the pathophysiology of autoimmunity. As ICIs have moved from the realm of clinical trials into the standard-of-care space, we are seeing reports of patients with preexisting autoimmunity who do in fact develop exacerbations with these treatments. $^{72,76-80}$ However, there have recently been two retrospective reviews of patients with autoimmune diseases receiving ICI treatment which have each reported similar and somewhat surprising findings. ${ }^{82,83}$ In a series of 30 patients with autoimmunity at baseline who received ipilimumab, $27 \%$ experienced a flare of their underlying autoimmunity and $33 \%$ experienced an irAE of $\geq$ grade 3 not related to their baseline autoimmune condition including one colitisrelated death; three patients fell into both categories. They describe 15 patients (50\%), however, who experienced neither an autoimmune flare nor a grade 3-or-higher event. Overall, $20 \%$ of the patients that Johnson et al reviewed had an objective response to ipilimumab treatment regardless of whether or not their underlying autoimmune condition flared. ${ }^{82}$ In another series describing pembrolizumab treatment in 52 patients with baseline autoimmune disease, 38\% of patients experienced a flare (with 17 of these 20 flares 
being grade 1-2), and $29 \%$ of patients experienced other irAEs (10 of these 15 events were also grade 1-2). Overall, $33 \%$ of patients reviewed by Menzies et al had an objective response to anti-PD-1 treatment regardless of the condition of their autoimmune disease activity. ${ }^{83}$ In one rare report, a patient receiving ipilimumab actually experienced a decrease in preexisting colitis symptoms during treatment. ${ }^{84}$ In another unique report, Uemura et al describe treatment of a patient with metastatic melanoma and a history of severe Crohn's colitis with simultaneous pembrolizumab and tocilizumab, transitioned later to pembrolizumab and adalimumab. The patient had a clinical complete response of her metastatic melanoma without clinical symptoms of Crohn's disease. ${ }^{85}$ Although limited in the sense that these series and reports are retrospective in nature and constitute a small number of cases, this evidence suggests that preexisting autoimmunity is not an absolute contraindication to treatment with ICIs, especially since many of these patients received benefit from treatment and reported durable antitumor effects. ${ }^{82,83}$

\section{irAE correlation with tumor response}

It has been hypothesized that an irAE may be a positive predictor of treatment response related to the mechanism of action of the ICI and its ability to fuel an immune response in the patient, but research into this area has had mixed conclusions. One group reports that the occurrence of an irAE of any severity or an irAE requiring corticosteroid treatment did not affect overall survival or time to treatment failure in their retrospectively analyzed cohort of melanoma patients treated with ipilimumab. ${ }^{51}$ Freeman-Keller et al, however, report that in their analysis of patients on trial with nivolumab and peptide vaccines for resected or unresectable melanoma, the occurrence of irAE of any grade, the occurrence of rash, or the occurrence of vitiligo was associated with an increased overall survival as compared to groups without these findings. ${ }^{86}$ In yet another study, Sanlorenzo et al report that cutaneous adverse events secondary to pembrolizumab were associated with an increased progression-free interval. The group also noted that hypopigmentation events in their cohort were only identified in melanoma patients, and, of the seven such events observed, only one patient's tumor progressed. ${ }^{87} \mathrm{~A}$ prospective study specifically focusing on vitiligo in melanoma patients treated with pembrolizumab reported an objective response rate of $71 \%$ in the group experiencing vitiligo as compared to a $28 \%$ objective response rate among those who did not develop vitiligo. This group pointed out that their detection of vitiligo in $25 \%$ of their pembrolizumab-treated melanoma patients, which is higher than reported anywhere else thus far, was most likely due to their thorough, prospective examinations of their patients specifically for this finding. ${ }^{88}$ More exploration of the theory that irAEs could be correlated with treatment response is warranted, but there are limitations to this analysis, which will be discussed below.

\section{Etiology of irAEs revisited}

The antigenicity of certain melanoma-associated proteins and the presence of autoantibodies to these proteins in the serum of melanoma patients have been described prior to the development of the ICI class, as has the positive prognostic value of hypopigmentation in melanoma patients. ${ }^{89-91}$ Perhaps it is safe to assume that ICIs enhance the antitumor immune response indicated by autoantibody presence, and that crossreaction of the neoantigen-directed antitumor response with normal melanocyte constituent proteins is the etiology behind the characteristic hypopigmentation. If vitiligo in melanoma patients first described decades ago is the same reaction that ICIs are potentiating today, then the observed effect would be an enhanced antitumor response and an apparent positive correlation between the irAE of vitiligo and the tumor response due to this cross-reactivity. A similar effect with a slightly different tumor and cross-reactive target could explain the observation that cutaneous irAEs in melanoma patients other than vitiligo also appear to be correlated with improved outcomes. In fact, the Sanlorenzo group's study, which correlated cutaneous adverse events with an increased progression-free interval, comprised $80 \%$ melanoma patients. ${ }^{86-88}$ Johnson et al explored this cross-reactivity theory of the etiology of irAEs in their description of two patients receiving the combination of ipilimumab and nivolumab who presented with fatal fulminant myocarditis and rhabdomyolysis. They describe a thorough postmortem evaluation of both patients' tumor, cardiac, and skeletal muscle tissues wherein they were able to demonstrate clonal expansions of T-cells in all three tissues of both patients. The theory proposed by this group is that one or more targets of each of the patients' antitumor immune responses were shared or very similar to antigens also expressed normally in skeletal and cardiac muscle. ${ }^{92}$ This theory is emphasized and supported by a short review of the Johnson group's work where Cheng and Loscalzo suggest that the cardiac irAEs observed in the Johnson report are due to low selectivity among the tumor-reactive T-cell population, and thus cross-reactivity with normal tissues. ${ }^{93}$

When all of the above evidence is considered, it suggests that irAEs are actually due to two separate etiologic pathways, 
as illustrated in Figure 1. First, the aforementioned hypothesis of preexisting tolerated self-reactive T-cells being deregulated in the periphery, and second, the theory of cross-reactivity between the target of an individual patient's antitumor immune response and normal tissues which share these same or similar targets. If this paradigm is correct and irAEs are in fact due to two different etiologies, then any investigation into their correlation with outcomes or the ability to predict these events is potentially confounded by this biologic difference.

For the purposes of predicting adverse events, it may be possible to relate irAEs due to tumor-independent autoreactive T-cells with preexisting biomarkers such as high-risk HLA haplotypes or serum screening for low-level detectable autoantibodies. Indeed, investigators have proposed various strategies to this end, but data on the validity and feasibility of these strategies are not available as of yet. ${ }^{94}$ Predictive biomarkers for cross-reactive irAEs between a patient's tumor and the patient's normal physiologic constituents may be more difficult to identify as each patient's tumor will produce its own complement of neoantigens. On the other hand, a biomarker that is independent of the etiology of an irAE would have greater utility and flexibility in clinical practice as it would in theory not suffer from this confounder or from the need to be patient-specific or tumor-specific. A recent study out of Japan has proposed the possibility of using routine blood counts as a biomarker of grade $3 / 4$ or lung and gastrointestinal irAEs based on univariate and multivariate analysis of fluctuations in blood counts in melanoma patients receiving nivolumab. ${ }^{95}$ This strategy may have merit as an etiology-independent, nonspecific sign of irAEs. However, the multicenter Japanese study focused on changes in blood counts that occurred during an event as compared to baseline measures before treatment. Much more investigation is needed to establish the viability of this method for predictive use prior to the development of a severe event as well as whether this method is generalizable to a wider patient population receiving nivolumab for other malignancies or receiving other ICIs.

Two independent pathways for the development of irAEs would also explain the discordant results of investigations into correlation of irAEs and tumor response. The group of adverse events related to preexisting autoreactive T-cells would likely not be correlated with tumor response, thus confounding the results of correlation studies which included all irAEs such as the Horvat group's study. ${ }^{51}$ The correlation signal that starts to appear when these studies analyze more specific events, such as cutaneous irAEs or vitiligo specifically in melanoma patients, may be due to the shift in etiologies of the irAEs being captured from a mixture of the first and second pathways to mostly the second or cross-reactivity pathway.

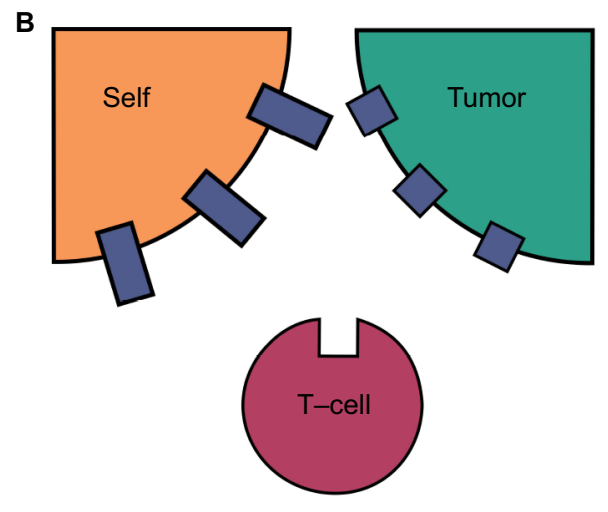

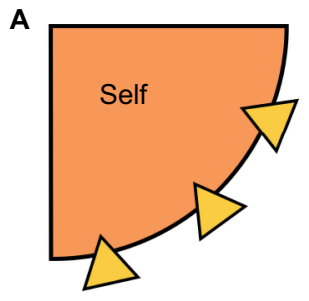
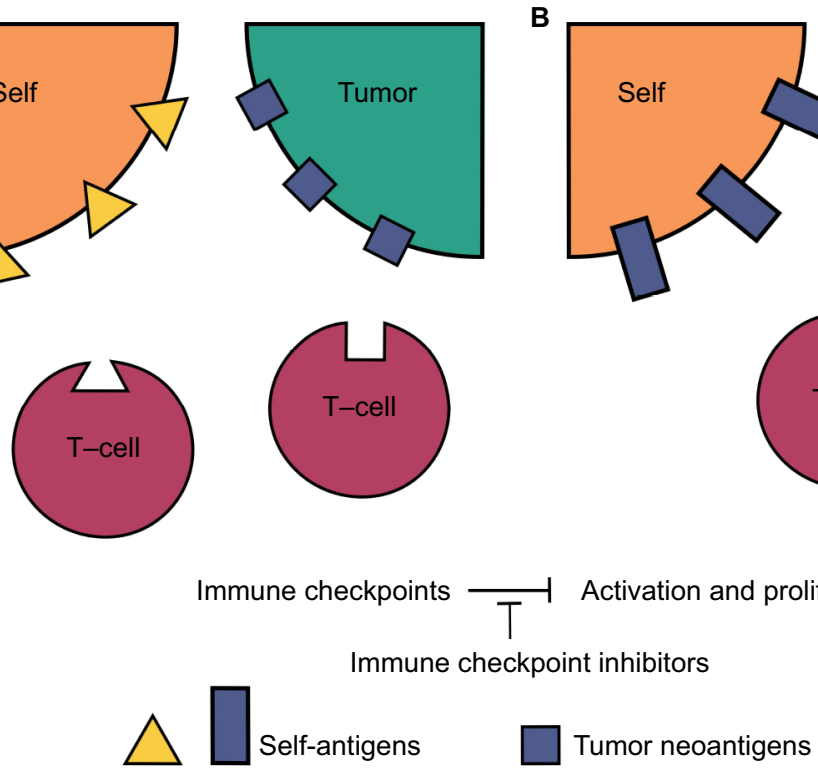

Figure I Peripherally tolerized T-cells and their target antigens in irAEs.

Notes: In both tumor environments, neoantigens caused an anti-tumor immune response, which has subsequently been downregulated through immune checkpoint pathways. (A) Self-reactive peripheral T-cells have also been tolerized through immune checkpoints. Treatment with immune checkpoint inhibitors in this environment will lead to activation and upregulation of both the anti-tumor and an autoimmune reaction. If the tumor-specific response is weak or nonexistent in this setting, the autoimmune irAE will appear in isolation without a corresponding tumor response. (B) The tumor-reactive tolerized T-cell not only displays affinity for tumor neoantigens, but also for similar self-antigens on healthy tissue. Immune checkpoint inhibitor treatment in this environment will lead to a cross-reactivity irAE in addition to a tumor-specific response. If the tumor-specific response is weaker than the cross-reactive response, it may also appear that and irAE has occurred in isolation in this setting. Abbreviation: irAE, immune-related adverse event. 
Further research into the area of irAEs should focus on the separation of events by etiology so that they can be more accurately predicted, treated, and correlated with outcomes.

\section{Conclusion}

With the recent paradigm shift in cancer treatment toward the harnessing of the immune system, there has been a concomitant shift in the types of side effects observed with cancer treatment. Although a majority of irAEs arising from PD-1, PD-L1, and CTLA-4 blockade appear to be of mild to moderate severity and responsive to corticosteroids or other systemic immunosuppressants, at times these events can be serious, irreversible, or even fatal. For now, the best defense against these irAEs appears to be knowledge of their existence, careful surveillance, and a low threshold for initiating or escalating immunosuppressant treatments. ${ }^{51}$ As novel ICIs and their combinations move through clinical trials and gain approval for use in patients with various malignancies and as approved ICIs enter clinical trials for the treatment of a variety of chronic infectious diseases, the issue of irAEs will continue to be an area of active investigation. Researchers and clinicians should focus future efforts on clarifying the pathogenesis of these events in order to reduce the confounding effects that may currently be present in the literature, with the ultimate goal of using this powerful new tool in the safest and most effective way possible.

\section{Disclosure}

The author reports no conflicts of interest in this work.

\section{References}

1. Pedoeem A, Azoulay-Alfaguter I, Strazza M, Silverman GJ, Mor A. Programmed death-1 pathway in cancer and autoimmunity. Clin Immunol. 2014;153(1):145-152.

2. Dyck L, Mills KHG. Immune checkpoints and their inhibition in cancer and infectious diseases. Eur J Immunol. 2017;47(5):765-779.

3. Rao M, Valentini D, Dodoo E, Zumla A, Maeurer M. Anti-PD-1/PD-L1 therapy for infectious diseases: learning from the cancer paradigm. Int J Infect Dis. 2017;56:221-228.

4. Tivol EA, Borriello F, Schweitzer AN, Lynch WP, Bluestone JA, Sharpe AH. Loss of CTLA-4 leads to massive lymphoproliferation and fatal multiorgan tissue destruction, revealing a critical negative regulatory role of CTLA-4. Immunity. 1995;3(5):541-547.

5. Weber J. Immune checkpoint proteins: a new therapeutic paradigm for cancer - preclinical background: CTLA-4 and PD-1 blockade. Semin Oncol. 2010;37(5):430-439.

6. Keir ME, Liang SC, Guleria I, et al. Tissue expression of PD-L1 mediates peripheral T cell tolerance. J Exp Med. 2006;203(4):883-895.

7. Ribas A. Adaptive immune resistance: how cancer protects from immune attack. Cancer Discov. 2015;5(9):915-919.

8. Weintraub K. Drug development: releasing the brakes. Nature. 2013;504(7480):S6-S8.

9. FDA.Drugs@FDA: FDA approved drug products. 2014. Available from: http//www.accessdata.fda.gov/ scripts/cder/daf. Accessed September $14,2017$.
10. FDA. FDA approves first cancer treatment for any solid tumor with a specific genetic feature. 2017. Available from: https://www.fda.gov/ newsevents/newsroom/pressannouncements/ucm560167.htm. Accessed September 14, 2017.

11. Okazaki T, Tanaka Y, Nishio R, et al. Autoantibodies against cardiac troponin I are responsible for dilated cardiomyopathy in PD-1-deficient mice. Nat Med. 2003;9(12):1477-1483.

12. Nishimura H, Nose M, Hiai H, Minato N, Honjo T. Development of lupus-like autoimmune diseases by disruption of the PD-1 gene encoding an ITIM motif-carrying immunoreceptor. Immunity. 1999;11: 141-151.

13. Wolchok JD, Neyns B, Linette G, et al. Ipilimumab monotherapy in patients with pretreated advanced melanoma: a randomised, double-blind, multicentre, phase 2, dose-ranging study. Lancet Oncol. 2010;11(2):155-164.

14. Topalian SL, Hodi FS, Brahmer JR, et al. Safety, activity, and immune correlates of anti-PD-1 antibody in cancer. $N$ Engl J Med. 2012;366(26):2443-2454

15. Weber JS, O'Day S, Urba W, et al. Phase I/II study of ipilimumab for patients with metastatic melanoma. J Clin Oncol. 2008;26(36): 5950-5956.

16. Brahmer JR, Drake CG, Wollner I, et al. Phase I study of single-agent anti-programmed death-1 (MDX-1106) in refractory solid tumors: safety, clinical activity, pharmacodynamics, and immunologic correlates. J Clin Oncol. 2010;28(19):3167-3175.

17. Hamid O, Robert C, Daud A, et al. Safety and tumor responses with lambrolizumab (anti-PD-1) in melanoma. $N$ Engl J Med. 2013;369(2):134-144.

18. Patnaik A, Kang SP, Rasco D, et al. Phase I study of pembrolizumab (MK-3475; anti-PD-1 monoclonal antibody) in patients with advanced solid tumors. Clin Cancer Res. 2015;21(19):4286-4293.

19. Robert C, Ribas A, Wolchok JD, et al. Anti-programmed-death-receptor-1 treatment with pembrolizumab in ipilimumab-refractory advanced melanoma: a randomised dose-comparison cohort of a phase 1 trial. Lancet. 2014;384(9948):1109-1117.

20. Day D, Hansen AR. Immune-related adverse events associated with immune checkpoint inhibitors. BioDrugs. 2016;30(6):571-584.

21. Haanen JBAG, van Thienen $H$, Blank CU. Toxicity patterns with immunomodulating antibodies and their combinations. Semin Oncol. 2015;42(3):423-428.

22. Wolchok JD, Kluger H, Callahan MK, et al. Nivolumab plus ipilimumab in advanced melanoma. N Engl J Med. 2013;369(2):122-133.

23. Antonia S, Goldberg SB, Balmanoukian A, et al. Safety and antitumour activity of durvalumab plus tremelimumab in non-small cell lung cancer: a multicentre, phase 1b study. Lancet Oncol. 2016;17(3):299-308.

24. Postow MA, Chesney J, Pavlick AC, et al. Nivolumab and ipilimumab versus ipilimumab in untreated melanoma. $N$ Engl J Med. 2015;372(21):2006-2017.

25. Levine JJ, Somer RA, Hosoya H, Squillante C. Atezolizumab-induced encephalitis in metastatic bladder cancer: a case report and review of the literature. Clin Genitourin Cancer. Epub 2017 Mar 16.

26. Hickmott L, De La Peña H, Turner H, et al. Anti-PD-L1 atezolizumabinduced autoimmune diabetes: a case report and review of the literature. Target Oncol. 2017;12(2):235-241.

27. Carrera W, Baartman BJ, Kosmorsky G. A case report of drug-induced myopathy involving extraocular muscles after combination therapy with tremelimumab and durvalumab for non-small cell lung cancer. Neuroophthalmology. 2017;41(3):140-143.

28. Rosenberg JE, Hoffman-Censits J, Powles T, et al. Atezolizumab in patients with locally advanced and metastatic urothelial carcinoma who have progressed following treatment with platinum-based chemotherapy: a single-arm, multicentre, phase 2 trial. Lancet. 2016;387(10031): 1909-1920.

29. Massard C, Gordon MS, Sharma S, et al. Safety and efficacy of durvalumab (MEDI4736), an anti-programmed cell death ligand-1 immune checkpoint inhibitor, in patients with advanced urothelial bladder cancer. J Clin Oncol. 2016;34(26):3119-3125. 
30. Kaufman HL, Russell J, Hamid O, et al. Avelumab in patients with chemotherapy-refractory metastatic Merkel cell carcinoma: a multicentre, single-group, open-label, phase 2 trial. Lancet Oncol. 2016;17(10):1374-1385.

31. Gulley JL, Rajan A, Spigel DR, et al. Avelumab for patients with previously treated metastatic or recurrent non-small-cell lung cancer (JAVELIN solid tumor): dose-expansion cohort of a multicentre, openlabel, phase 1b trial. Lancet Oncol. 2017;18(5):599-610.

32. Heery CR, O'Sullivan-Coyne G, Madan RA, et al. Avelumab for metastatic or locally advanced previously treated solid tumours (JAVELIN solid tumor): a phase 1a, multicohort, dose-escalation trial. Lancet Oncol. 2017;18(5):587-598.

33. Apolo AB, Infante JR, Balmanoukian A, et al. Avelumab, an antiprogrammed death-ligand 1 antibody, in patients with refractory metastatic urothelial carcinoma: results from a multicenter, phase $\mathrm{Ib}$ study. J Clin Oncol. 2017;35(19):2117-2124.

34. McDermott DF, Sosman JA, Sznol M, et al. Atezolizumab, an antiprogrammed death-ligand 1 antibody, in metastatic renal cell carcinoma: long-term safety, clinical activity, and immune correlates from a phase Ia study. J Clin Oncol. 2016;34(8):833-842.

35. Herbst RS, Soria J-C, Kowanetz M, et al. Predictive correlates of response to the anti-PD-L1 antibody MPDL3280A in cancer patients. Nature. 2014;515(7528):563-567.

36. Abdel-Wahab N, Shah M, Suarez-Almazor ME. Adverse events associated with immune checkpoint blockade in patients with cancer: a systematic review of case reports. PLoS One. 2016;11(7): e0160221.

37. Aya F, Ruiz-Esquide V, Viladot M, et al. Vasculitic neuropathy induced by pembrolizumab. Ann Oncol. 2017;28(2):433-434.

38. de Velasco G, Bermas B, Choueiri TK. Autoimmune arthropathy and uveitis as complications of programmed death 1 inhibitor treatment. Arthritis Rheumatol. 2016;68(2):556-557.

39. Gelsomino F, Vitale G, D’Errico A, Bertuzzi C, Andreone P, Ardizzoni A. Nivolumab-induced cholangitic liver disease: a novel form of serious liver injury. Ann Oncol. 2017;28(3):671-672.

40. Jung K, Zeng X, Bilusic M. Nivolumab-associated acute glomerulonephritis: a case report and literature review. BMC Nephrol. 2016;17(1):188

41. Khokhar MO, Kettle J, Palla AR. Debilitating skin toxicity associated with pembrolizumab therapy in an 81-year-old female with malignant melanoma. Case Rep Oncol. 2016;9(3):833-839.

42. Larsabal M, Marti A, Jacquemin C, et al. Vitiligo-like lesions occurring in patients receiving anti-programmed cell death-1 therapies are clinically and biologically distinct from vitiligo. J Am Acad Dermatol. 2017;76(5):863-870.

43. Läubli H, Balmelli C, Bossard M, Pfister O, Glatz K, Zippelius A. Acute heart failure due to autoimmune myocarditis under pembrolizumab treatment for metastatic melanoma. J Immunother Cancer. 2015;3(1):11.

44. Ohtsuka M, Miura T, Mori T, Ishikawa M, Yamamoto T. Occurrence of psoriasiform eruption during nivolumab therapy for primary oral mucosal melanoma. JAMA Dermatol. 2015;151(7):797.

45. Semper H, Muehlberg F, Schulz-Menger J, Allewelt M, Grohé C. Drug-induced myocarditis after nivolumab treatment in a patient with PDL1-negative squamous cell carcinoma of the lung. Lung Cancer. 2016;99:117-119.

46. Simonelli M, Di Tommaso L, Baretti M, Santoro A. Pathological characterization of nivolumab-related liver injury in a patient with glioblastoma. Immunotherapy. 2016;8(12):1363-1369.

47. Tanaka R, Maruyama H, Tomidokoro Y, et al. Nivolumab-induced chronic inflammatory demyelinating polyradiculoneuropathy mimicking rapid-onset Guillain-Barre syndrome: a case report. Jpn J Clin Oncol. 2016;46(9):875-878.

48. Tardy MP, Gastaud L, Boscagli A, Peyrade F, Gallamini A, Thyss A. Autoimmune hemolytic anemia after nivolumab treatment in Hodgkin lymphoma responsive to immunosuppressive treatment. A case report. Hematol Oncol. Epub 2016 Aug 19.
49. Cappelli LC, Gutierrez AK, Baer AN, et al. Inflammatory arthritis and sicca syndrome induced by nivolumab and ipilimumab. Ann Rheum Dis. 2017;76(1):43-50.

50. Hofmann L, Forschner A, Loquai C, et al. Cutaneous, gastrointestinal, hepatic, endocrine, and renal side-effects of anti-PD-1 therapy. Eur $J$ Cancer. 2016;60:190-209.

51. Horvat TZ, Adel NG, Dang TO, et al. Immune-related adverse events, need for systemic immunosuppression, and effects on survival and time to treatment failure in patients with melanoma treated with ipilimumab at Memorial Sloan Kettering Cancer Center. J Clin Oncol. 2015;33(28):3193-3198.

52. Aleksova J, Lau PKH, Soldatos G, McArthur G. Glucocorticoids did not reverse type 1 diabetes mellitus secondary to pembrolizumab in a patient with metastatic melanoma. BMJ Case Rep. Epub 2016 Nov 23.

53. Miyoshi Y, Ogawa O, Oyama Y. Nivolumab, an anti-programmed cell death-1 antibody, induces fulminant type 1 diabetes. Tohoku J Exp Med. 2016;239(2):155-158

54. Martin-Liberal J, Furness AJ, Joshi K, Peggs KS, Quezada SA, Larkin J. Anti-programmed cell death-1 therapy and insulin-dependent diabetes: a case report. Cancer Immunol Immunother. 2015;64(6):765-767.

55. Hughes J, Vudattu N, Sznol M, et al. Precipitation of autoimmune diabetes with anti-PD-1 immunotherapy. Diabetes Care. 2015;38(4):e55-e57.

56. Trainer H, Hulse P, Higham CE, Trainer P, Lorigan P. Hyponatraemia secondary to nivolumab-induced primary adrenal failure. Endocrinol Diabetes Metab Case Rep. Epub 2016 Nov 1.

57. Ryder M, Callahan M, Postow MA, Wolchok J, Fagin JA. Endocrinerelated adverse events following ipilimumab in patients with advanced melanoma: a comprehensive retrospective review from a single institution. Endocr Relat Cancer. 2014;21(2):371-381.

58. Ong M, Ibrahim AM, Bourassa-Blanchette S, Canil C, Fairhead T, Knoll G. Antitumor activity of nivolumab on hemodialysis after renal allograft rejection. J Immunother Cancer. 2016;4(1):64.

59. Spain L, Higgins R, Gopalakrishnan K, Turajlic S, Gore M, Larkin $\mathrm{J}$. Acute renal allograft rejection after immune checkpoint inhibitor therapy for metastatic melanoma. Ann Oncol. 2016;27(6):1135-1137.

60. Lipson EJ, Bagnasco SM, Moore J, et al. Tumor regression and allograft rejection after administration of anti-PD-1. $N$ Engl J Med. 2016;374(9):896-898.

61. Barnett R, Barta VS, Jhaveri KD. Preserved renal-allograft function and the PD-1 pathway inhibitor nivolumab. $N$ Engl J Med. 2017;376(2): 191-192.

62. Alhamad T, Venkatachalam K, Linette GP, Brennan DC. Checkpoint inhibitors in kidney transplant recipients and the potential risk of rejection. Am J Transplant. 2016;16(4):1332-1333.

63. Owonikoko TK, Kumar M, Yang S, et al. Cardiac allograft rejection as a complication of PD-1 checkpoint blockade for cancer immunotherapy: a case report. Cancer Immunol Immunother. 2017;66(1):45-50.

64. Wanchoo R, Riella L V, Uppal NN, et al. Immune checkpoint inhibitors in the cancer patient with an organ transplant. J Onco-Nephrol. 2017;1(1):42-48.

65. Behling J, Kaes J, Münzel T, Grabbe S, Loquai C. New-onset thirddegree atrioventricular block because of autoimmune-induced myositis under treatment with anti-programmed cell death-1 (nivolumab) for metastatic melanoma. Melanoma Res. 2017;27(2):155-158.

66. Gibson R, Delaune J, Szady A, Markham M. Suspected autoimmune myocarditis and cardiac conduction abnormalities with nivolumab therapy for non-small cell lung cancer. BMJ Case Rep. Epub 2016 Jul 20.

67. Palla AR, Kennedy D, Mosharraf H, Doll D. Autoimmune hemolytic anemia as a complication of nivolumab therapy. Case Rep Oncol. 2016;9(3):691-697.

68. Nishino M, Sholl LM, Hatabu H, Ramaiya NH, Hodi FS. Anti-PD1-related pneumonitis during cancer immunotherapy. $N$ Engl J Med. 2015;373(3):288-290.

69. Maurice C, Schneider R, Kiehl T, et al. Subacute CNS demyelination after treatment with nivolumab for melanoma. Cancer Immunol Res. 2015;3(12):1299-1302. 
70. Shirai T, Sano T, Kamijo F, et al. Acetylcholine receptor binding antibodyassociated myasthenia gravis and rhabdomyolysis induced by nivolumab in a patient with melanoma. Jpn J Clin Oncol. 2016;46(1):86-88.

71. Loochtan AI, Nickolich MS, Hobson-Webb LD. Myasthenia gravis associated with ipilimumab and nivolumab in the treatment of small cell lung cancer. Muscle Nerve. 2015;52(2):307-308.

72. Phadke SD, Ghabour R, Swick BL, SwensonA, Milhem M, Zakharia Y. Pembrolizumab therapy triggering an exacerbation of preexisting autoimmune disease. J Investig Med High Impact Case Rep. 2016;4(4):2324709616674316.

73. Kimura T, Fukushima S, Miyashita A, et al. Myasthenic crisis and polymyositis induced by one dose of nivolumab. Cancer Sci. 2016;107(7): 1055-1058.

74. Gonzalez NL, Puwanant A, Lu A, Marks SM, Živkovi SA. Myasthenia triggered by immune checkpoint inhibitors: new case and literature review. Neuromuscul Disord. 2017;27(3):266-268.

75. Kong BY, Micklethwaite KP, Swaminathan S, Kefford RF, Carlino MS. Autoimmune hemolytic anemia induced by anti-PD-1 therapy in metastatic melanoma. Melanoma Res. 2016;26(2):202-204.

76. Kato Y, Otsuka A, Miyachi Y, Kabashima K. Exacerbation of psoriasis vulgaris during nivolumab for oral mucosal melanoma. J Eur Acad Dermatol Venereol. 2016;30(10):e89-e91.

77. Narita T, Oiso N, Taketomo Y, et al. Serological aggravation of autoimmune thyroid disease in two cases receiving nivolumab. J Dermatol. 2016;43(2):210-214.

78. Cotliar J, Querfeld C, Boswell WJ, Raja N, Raz D, Chen R. Pembrolizumab-associated sarcoidosis. JAAD Case Rep. 2016;2(4):290-293.

79. Lau KHV, Kumar A, Yang IH, Nowak RJ. Exacerbation of myasthenia gravis in a patient with melanoma treated with pembrolizumab. Muscle Nerve. 2016;54(1):157-161.

80. Beck KM, Dong J, Geskin LJ, et al. Disease stabilization with pembrolizumab for metastatic acral melanoma in the setting of autoimmune bullous pemphigoid. J Immunother Cancer. 2016;4(1):20.

81. Beck KE, Blansfield JA, Tran KQ, et al. Enterocolitis in patients with cancer after antibody blockade of cytotoxic T-lymphocyte-associated antigen 4. J Clin Oncol. 2006;24(15):2283-2289.

82. Johnson DB, Sullivan RJ, Ott PA, et al. Ipilimumab therapy in patients with advanced melanoma and preexisting autoimmune disorders. JAMA Oncol. 2016;2(2):234.

83. Menzies AM, Johnson DB, Ramanujam S, et al. Anti-PD-1 therapy in patients with advanced melanoma and preexisting autoimmune disorders or major toxicity with ipilimumab. Ann Oncol. 2016;28(2):368-376.
84. Pedersen M, Andersen R, Nørgaard P, et al. Successful treatment with ipilimumab and interleukin-2 in two patients with metastatic melanoma and systemic autoimmune disease. Cancer Immunol Immunother. 2014;63(12):1341-1346.

85. Uemura M, Trinh VA, Haymaker C, et al. Selective inhibition of autoimmune exacerbation while preserving the anti-tumor clinical benefit using IL-6 blockade in a patient with advanced melanoma and Crohn's disease: a case report. J Hematol Oncol. 2016;9(1):81.

86. Freeman-Keller M, Kim Y, Cronin H, Richards A, Gibney G, Weber JS. Nivolumab in resected and unresectable metastatic melanoma: characteristics of immune-related adverse events and association with outcomes. Clin Cancer Res. 2016;22(4):886-894.

87. Sanlorenzo M, Vujic I, Daud A, et al. Pembrolizumab cutaneous adverse events and their association with disease progression. JAMA Dermatol. 2015;151(11):1206.

88. Hua C, Boussemart L, Mateus C, et al. Association of vitiligo with tumor response in patients with metastatic melanoma treated with pembrolizumab. JAMA Dermatol. 2016;152(1):45.

89. Huang SKS, Okamoto T, Morton DL, Hoon DSB. Antibody responses to melanoma/melanocyte autoantigens in melanoma patients. $J$ Invest Dermatol. 1998;111(4):662-667.

90. Nordlund J, Kirkwood J, Forget B, Milton G. Vitiligo in patients with metastatic melanoma: a good prognostic sign. J Am Acad Dermatol. 1983;9(5):689-696.

91. Bystryn JC, Rigel D, Friedman RJ, Kopf A. Prognostic significance of hypopigmentation in malignant melanoma. Arch Dermatol. 1987;123(8): 1053-1055.

92. Johnson DB, Balko JM, Compton ML, et al. Fulminant myocarditis with combination immune checkpoint blockade. NEngl J Med. 2016;375(18): 1749-1755.

93. Cheng F, Loscalzo J. Autoimmune cardiotoxicity of cancer immunotherapy. Trends Immunol. 2017;38(2):77-78.

94. Spain L, Larkin J, Martin-Liberal J. Determining predictive factors for immune checkpoint inhibitor toxicity: response to Letter to the Editors "A case report of insulin-dependent diabetes as immune-related toxicity of pembrolizumab: presentation, management and outcome." Cancer Immunol Immunother. 2016;65(6):769-770.

95. Fujisawa Y, Yoshino K, Otsuka A, et al. Fluctuations in routine blood count might signal severe immune-related adverse events in melanoma patients treated with nivolumab. J Dermatol Sci. Epub 2017 Jul 16.
ImmunoTargets and Therapy

\section{Publish your work in this journal}

ImmunoTargets and Therapy is an international, peer-reviewed open access journal focusing on the immunological basis of diseases, potential targets for immune based therapy and treatment protocols employed to improve patient management. Basic immunology and physiology of the immune system in health, and disease will be also covered. In addition, the journal will focus on the impact of manage-

\section{Dovepress}

ment programs and new therapeutic agents and protocols on patient perspectives such as quality of life, adherence and satisfaction. The manuscript management system is completely online and includes a very quick and fair peer-review system, which is all easy to use. Visit http://www.dovepress.com/testimonials.php to read real quotes from published authors. 